\title{
THE INTERNATIONAL ULTRAVIOLET EXPLORER
}

\section{Albert Boggess}

The International Ultraviolet Explorer is a new astronomical satellite still in the design stage. It is a Delta-launched satellite, weighing approximately 365 kilograms $(800 \mathrm{lb})$, with an intended lifetime of three to five years, and it will serve as a precursor to the Large Space Telescope. The essential features of the satellite are its geosynchronous orbit, a 45centimeter aperture telescope, and a high performance UV spectrograph using television tubes as detectors. It will be used as an international research facility, with NASA, ESRO, and the British Science Research Council each contributing to the hardware and participating in operation. Figure 1 shows an artist's concept of the satellite in orbit.

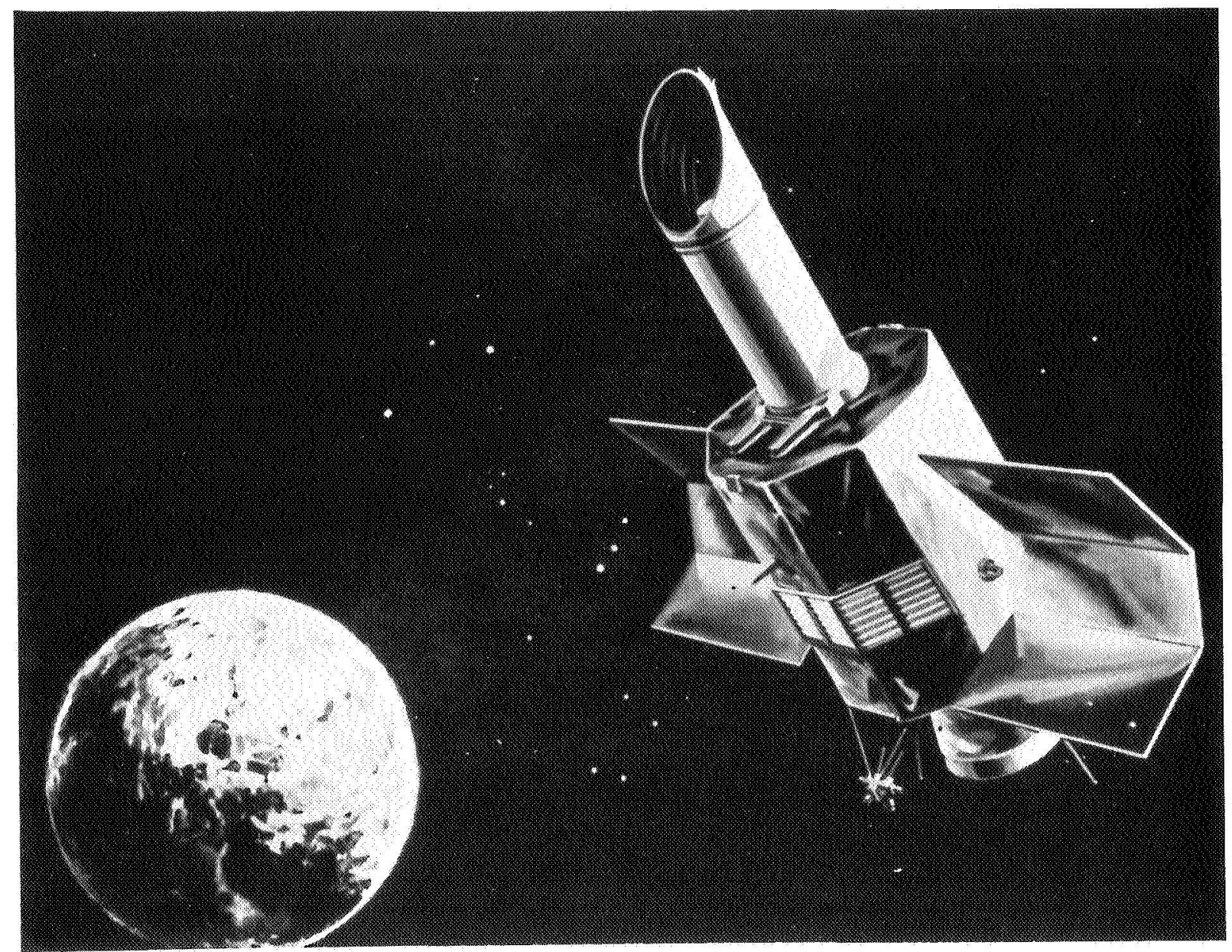

Figure 1. The IUE spacecraft. 
One can see the telescope tube sticking out of the satellite proper, with a cylindrical baffle on top. The primary mirror is located near the base of the tube. The solar arrays, which are to be provided by ESRO, are shown extended, and one sees at the back end the stationkeeping thrusters, antennae, and the apogee kick motor. Control is provided by a gyro package which, with periodic stellar updates, will be able to maintain inertial pointing to one second of arc for arbitrarily long periods of time. The satellite is to be stationed over the Atlantic, where it can be operated from ground stations either in the U.S. or in Europe. The anticipated usage is 16 hours per day from the U.S. and 8 hours per day controlled from Europe.

The optical system shown in Figure 2 includes a conventional Ritchey Chretien $\mathrm{f} / 15$ telescope, 45 centimeters in diameter. In the rear compartment are the spectrographs. I won't try to go through the complicated optical system in detail. There are two echelle spectrographs packaged side-by-side which provide a total wavelength coverage from 1150 to 3200 Angstroms at either high dispersion (0.1 Angstrom resolution) or low dispersion (6 Angstrom resolution). The large masses in the spectrograph cavity are the television cameras. A total of three tubes is required to record the spectra plus various auxiliary data. Each of these tubes has a spare, so that there is a total of six TV systems on board the spacecraft.

The spectrum detectors must record an image like that shown in Figure 3. Here we see a spectrum broken into short segments, starting at 1150 Angstroms down at the bottom and increasing from left to right at a dispersion of about one Angstrom per millimeter. The bottom order is only about a millimeter long, but the spectrum then picks up on the next line on the left and continues on, order by order, all the way to the long wavelength limit. The variations in density are due to spectral lines, and the job of the TV

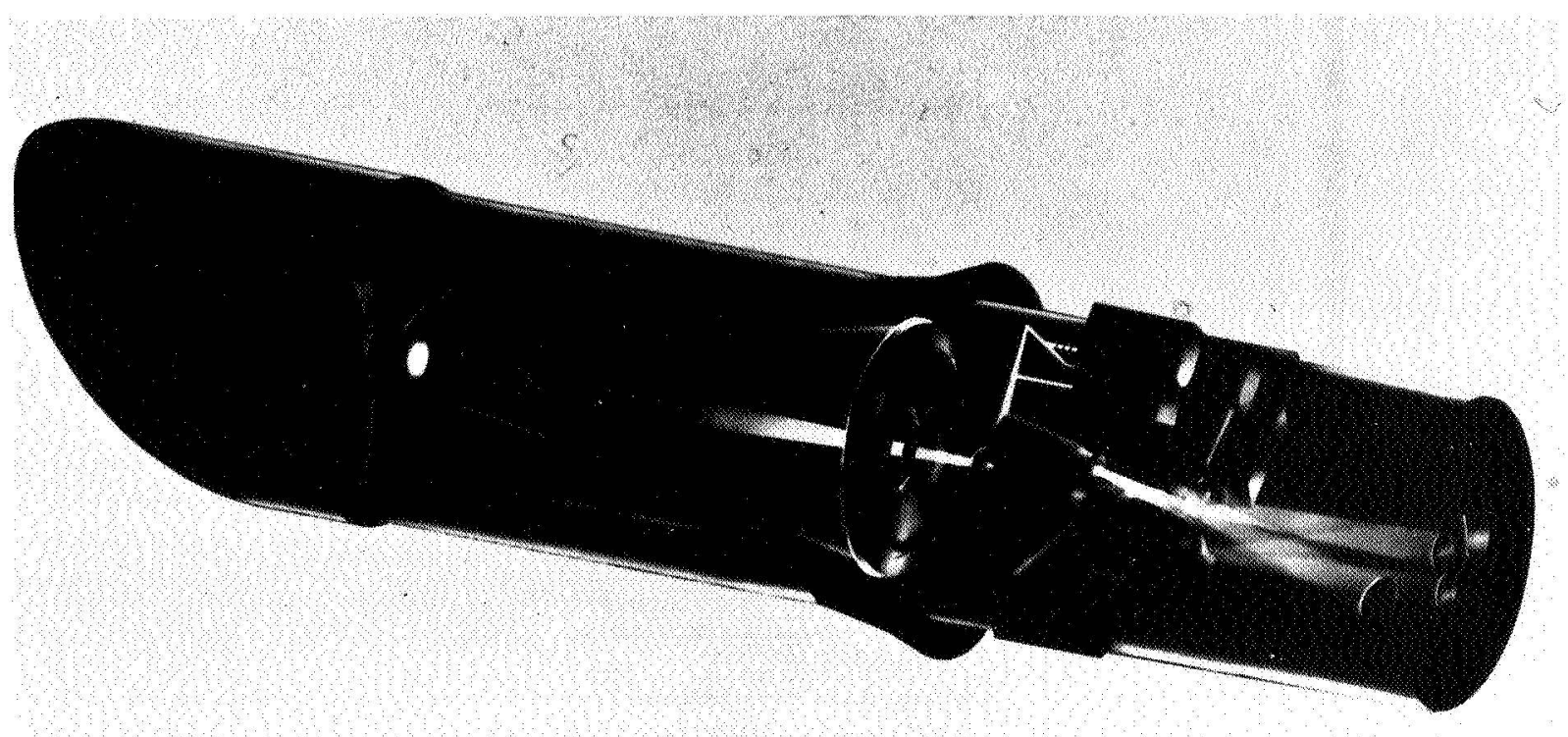

Figure 2. IUE scientific instrument. 
tube is to record the positions, e.g. the wavelengths, and the photometric profiles of these various features.

At the bottom of the picture, where the information density is greatest, the format corresponds to a bar chart with a spacing of 5 line pairs per millimeter, but the resolution of the tube must be greater than this implies. It should be greater because accurate photometric measurements must be obtained both in the spectrum and in the background between orders in order to make stray light corrections to the data.

The detector system that we have developed for this purpose includes the Westinghouse WL 30893 electrostatically focused SEC Vidicon, operated digitally. This tube meets the rather severe weight and volume requirements imposed on the system, but has a fiber optic faceplate which is able to transmit only visible light. In order to make this tube UVsensitive, it is coupled with a proximity focused photon converter with a cesium telluride photocathode.

The combination, then, the photon converter plus the SEC Vidicon, gives us an ultraviolet detector system which is capable of recording spectra such as these with a photometric statistical accuracy of some 2 percent.

The schedule requires that this system be flown about four years from now. At that time, it will present the highest capability astronomical research facility in orbit; and that will continue to be the case until the launch of the LST itself.

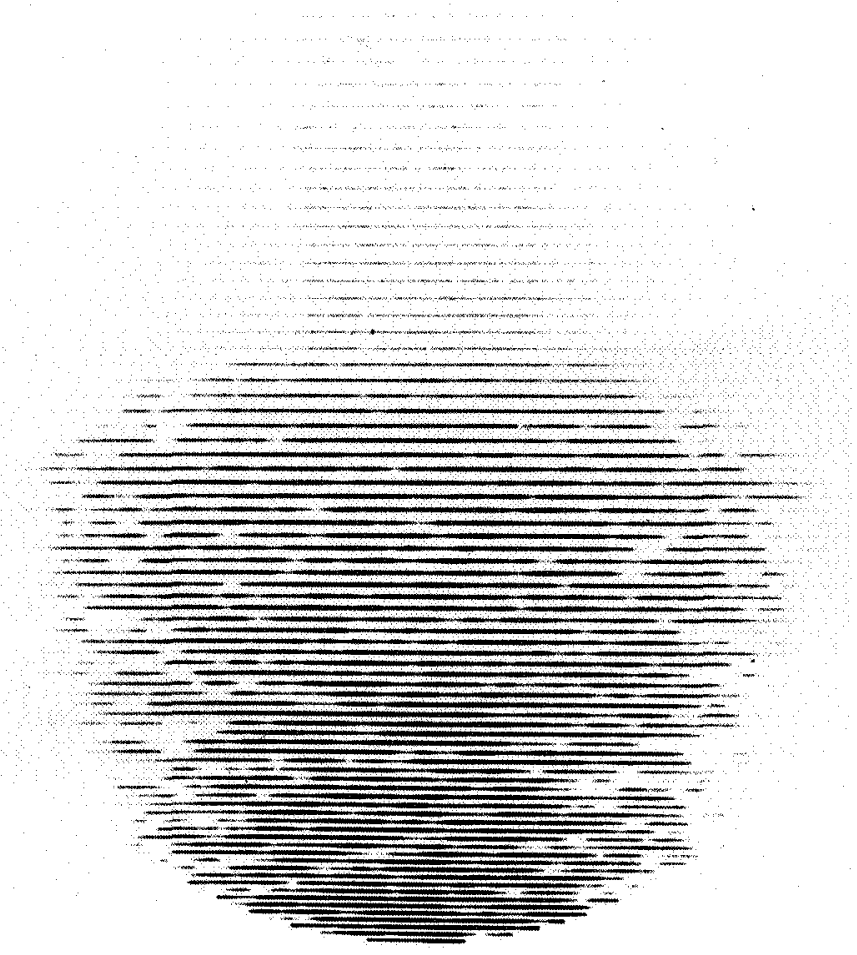

Figure 3. An Echelle spectrogram. 